\title{
Recubrimientos de materiales compuestos metal-cerámico obtenidos por nuevas tecnologías de proyección témica: Proyección fría (CGS) y su resistencia al desgaste ${ }^{(\cdot)}$
}

\author{
J. M. Miguel*, S. Vizcaíno*, S. Dosta*, N. Cinca*, C. Lorenzana* y J. M. Guilemany*
}

\begin{abstract}
Resumen En este artículo se utiliza la técnica de proyección fría (Cold Gas Spray -CGS-) para la obtención y caracterización de materiales compuestos constituidos por una matriz metálica de bronce de aluminio y una fase cerámica de alúmina con la intención de mejorar la resistencia al desgaste de los recubrimientos de bronce metálico puros. Se describen los diferentes procesos que ocurren durante la formación del recubrimiento (acritud de las partículas metálicas, rotura de las partículas cerámicas, efecto de granallado sobre el substrato metálico, entre otros) y se analizan cuáles son sus consecuencias sobre las propiedades del recubrimiento. Se han realizado ensayos de desgaste por fricción (ensayo Ball-on-Disk), abrasión (Rubber Wheel) y erosión, así como se ha determinado la microdureza y adherencia, y correlacionando los resultados obtenidos con el contenido de fase cerámica del recubrimiento. Se concluye que la fase cerámica reforzante incrementa sustancialmente las propiedades tribológicas con relación al bronce de partida. Finalmente se analizan los diferentes mecanismos de desgaste que tienen lugar durante los ensayos tribológicos.
\end{abstract}

Palabras clave

Proyección fría; Unión estado sólido; Tribología; Compuesto; Bronce; Alúmina; Refuerzo; Desgaste.

\section{Metal-ceramic composite coatings obtained by new thermal spray technologies: Cold Gas Spray (CGS) and its wear resistance}

\begin{abstract}
In this paper, composite coatings composed by an aluminum bronze metal matrix and a hard ceramic alumina phase obtained by cold spray technique were obtained in order to increase the tribological properties of the pure bronze coatings. The different processes that occur during the coating formation (hardening of the metal particles, fragmentation of the ceramic particles, shot peening on the metal substrate, etc) are described and their effects on the coating properties are studied. Wear tests consisting on Ball-on-Disk tests, abrasion Rubber Wheel tests and erosion tests as well as microhardness and adhesion tests are carried out and the results are correlated with the ceramic phase content of the coatings. It can be concluded that the hard ceramic phase increases the tribological properties with relation of the initial bronze coating. Finally, main wear mechanisms during the tribological tests are described.
\end{abstract}

Keywords

Cold spray; Solid state joining; Tribology; Composite; Bronze; Alumina; Reinforcement; Wear.

\section{INTRODUCCIÓN}

\subsection{Sobre el estado del arte de la proyección fría}

En los últimos años, las tecnologías de proyección térmica han evolucionado generando nuevos procedimientos y materiales que permiten realizar recubrimientos a medida para diferentes aplicaciones. Una de las limitaciones que existía en los procesos de proyección térmica convencionales (por ejemplo HVOF -High Velocity Oxy Fuel-, Plasma o llama) parece ser superado por un nuevo proceso denominado proyección fría (Cold Gas Spray, CGS). La proyección fría es un proceso de proyección en estado sólido, lo que hace posible la deposición de materiales susceptibles a la oxidación como son metales reactivos como el aluminio, cobre o titanio o materiales sensibles a la temperatura como es el caso de polvos nanoestructurados o amorfos ${ }^{[1-4]}$.

\footnotetext{
(•) Trabajo recibido el día 02 de agosto de 2010 y aceptado en su forma final, el día 11 de mayo de 2011.

* Centro de Proyección Térmica (CPT), Universidad de Barcelona, c/ Martí i Franqués 1, E-08028, Barcelona. Email: chemarimiguel@hotmail.com, Tel. +34934021302, Fax. +34934021638
} 
En la proyección fría las partículas se aceleran mediante un flujo de gas precalentado a alta presión que pasa a través de una boquilla tipo DeLaval y finalmente impactan con el substrato. Bajo condiciones óptimas, las partículas impactan generando un recubrimiento denso, sin presencia de óxidos (Fig.1). El rango de velocidad de las partículas varía entre 200 y $1.200 \mathrm{~m} / \mathrm{s}$, dependiendo de la geometría de la boquilla, densidad, tamaño y forma de la partícula y de la presión y temperatura del gas de procesado. Para una deposición adecuada se requiere, dependiendo del material, una cierta velocidad mínima o crítica, el valor de la cual depende significativamente de las propiedades termo-mecánicas del polvo y del substrato. Por debajo de esta velocidad crítica, las partículas que impactan generan solamente una erosión del substrato ${ }^{[5}$ y 6$]$. Por encima, la deposición de las partículas mejora hasta un máximo que en algunos casos puede superar el $95 \%$.

El estado topográfico y temperatura del substrato son también parámetros de alta importancia en la deposición de los recubrimientos. No existe aún un conocimiento profundo sobre las razones exactas que hacen que las partículas se adhieran al substrato, aunque se conoce que la capacidad de deformación de las partículas proyectadas así como del substrato son de gran importancia a la hora de realizar el recubrimiento ${ }^{[7]}$. Durante los primeros años de desarrollo de esta nueva técnica a nivel industrial (no llega a los 10 años) solamente se realizaban recubrimientos metálicos sobre superficies metálicas, aunque más recientemente se han empezado a realizar recubrimientos cerámicos y compuestos. De esta manera, algunos autores han podido obtener recubrimientos de materiales cerámicos (caso del $\mathrm{TiO}_{2}$ ) o compuestos cerámicos-metal (WC-Co o $\mathrm{Cr}_{3} \mathrm{C}_{2}-\mathrm{NiCr}$ ) con un muy alto contenido en partículas cerámicas, a pesar de su mínima capacidad de deformación ${ }^{[8-10]}$.

La proyección fría es una técnica reciente que está madurando rápidamente en la que hay una serie de tópicos en los que ingenieros, científicos y tecnólogos de alrededor del mundo están trabajando particularmente:

- Mejor conocimiento de los procesos de proyección, incluyendo la formación del haz, la interacción de las partículas en el mismo, el impacto de las partículas y la constitución del recubrimiento, las propiedades del recubrimiento, etc. Existen numerosos trabajos intentando simular las condiciones de proyección y que tienen como finalidad una puesta a punto de condiciones de trabajo más fácil, sin tener que realizar ensayos previos.

- El desarrollo, diseño, ensayo y comercialización de nuevos equipos de proyección fría para un trabajo fácil, seguro y eficaz. De esta manera, aunque existen ya equipos de proyección fría portátiles, se están desarrollando nuevos sistemas que pueden trabajar en mejo-

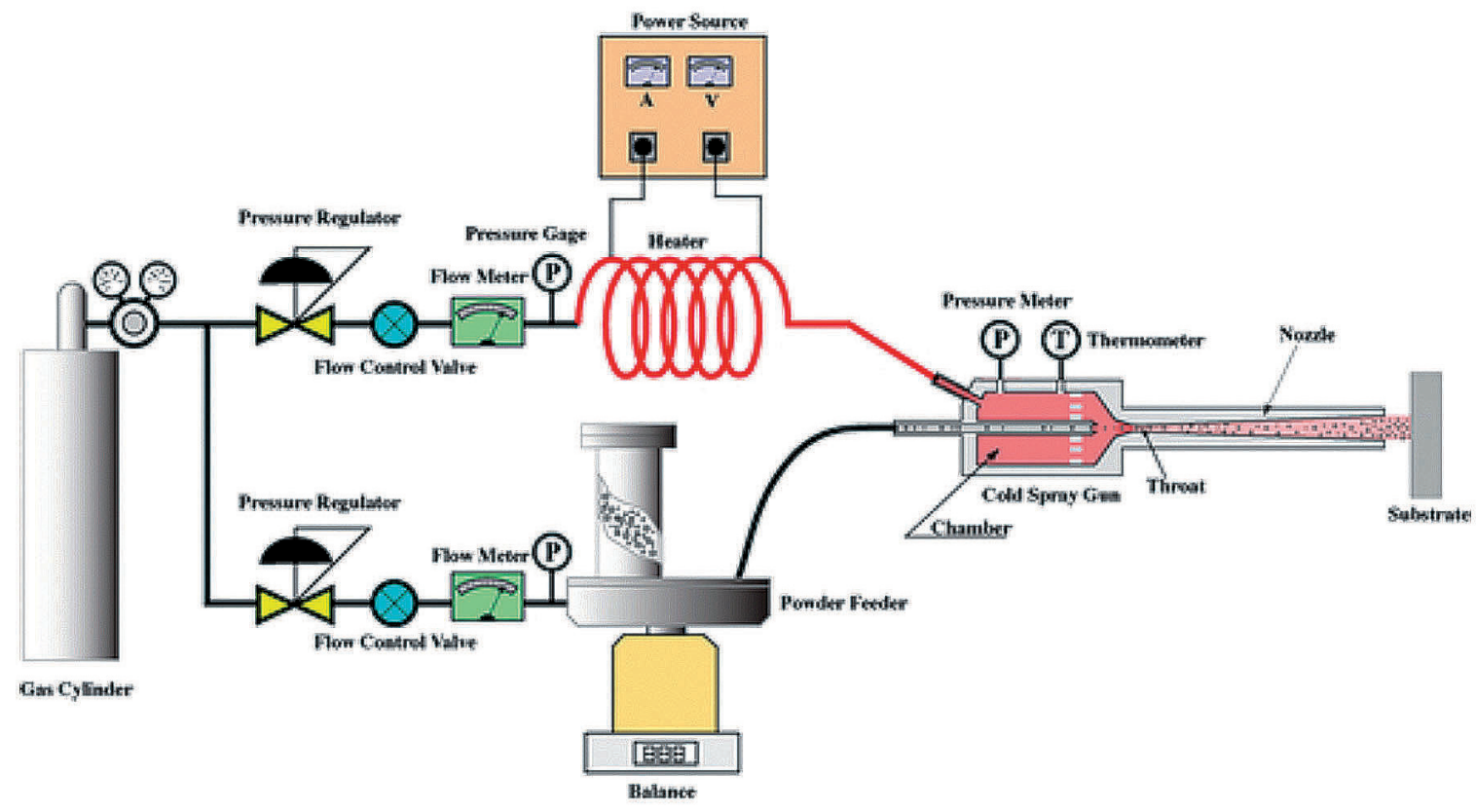

Figura 1. Sistema de proyección fría (CGS).

Figura 1. Cold spray technique (CGS). 
res condiciones y así producir recubrimientos con mejores propiedades. También se están desarrollando nuevas boquillas que permiten alcanzar velocidades de proyección superiores.

- Desarrollo de nuevos recubrimientos para diferentes aplicaciones. La proyección fría permite desarrollar capas de un grosor situado entre las decenas de micrómetros hasta los milímetros. La posible utilización de nuevos recubrimientos hasta ahora no proyectables han abierto las puertas a aplicaciones, como sería la deposición de capas de titanio biocompatible o aluminio 6061 con alta resistencia máxima para reparación de plataformas de vehículos.

- Adaptación industrial y ayuda a la comercialización de recubrimientos de proyección fría. Todo el esfuerzo aplicado al desarrollo de nuevos recubrimientos repercute en la comercialización y promoción de esta nueva tecnología.

La proyección fría es hoy una herramienta para la utilización industrial, aunque los límites de esta técnica están aún por descubrir.

\subsection{Sobre la situación de los recubrimientos obtenidos por proyección fría}

Mediante proyección fría se han conseguido realizar recubrimientos de materiales que hasta ahora presentaban una gran dificultad de obtención, especialmente el titanio o el cobre ${ }^{[11]}$. Es posible obtener recubrimientos de titanio totalmente densos, o por el contrario, recubrimientos con una alta porosidad, pudiéndose utilizar principalmente en implantes biomédicos o aplicaciones aeronáuticas. Los recubrimientos de aluminio obtenidos por esta técnica ya son utilizados para proteger o reparar componentes de aluminio, mientras que los compuestos cerámicometal tipo WC-CoCr (muy utilizados en proyección térmica convencional) tienen una importante aplicabilidad en condiciones de alto desgaste y/o corrosión como en válvulas, cilindros o ejes ${ }^{[12]}$.

El bronce es un material utilizado tradicionalmente por su buena resistencia al desgaste por fricción y su bajo coeficiente de deslizamiento, siendo utilizado, por ejemplo, en anillos de sincronización para la industria del automóvil ${ }^{[13}$ y 14]. En algunos casos su buena resistencia a la corrosión y correcto comportamiento a elevadas temperaturas le permite ser utilizado en husillos de válvulas, ejes para mecanismos agitadores, guías, engranajes y piezas de forja.
En la familia de materiales tipo bronce, los bronces de aluminio son aleaciones especialmente utilizadas por su mayor resistencia al desgaste. Este grupo está formado por aleaciones conteniendo hasta $12 \%$ en peso de aluminio, con adiciones de hierro, níquel y manganeso. Estas aleaciones alcanzan valores de dureza del rango de 250-400 HB, incrementándose al aumentar el contenido de aluminio, aunque como contrapartida baja su ductilidad ${ }^{[15]}$. De esta manera, estos materiales pueden utilizarse en su composición de más alta dureza para aplicaciones como herramientas de embutición profunda, herramientas de doblado y moldes donde se requiera una alta resistencia al desgaste, entre otras.

La proyección fría es una tecnología que permite utilizar polvos de bronce de aluminio para hacer reparaciones de herramientas de composición similar o recubrimientos sobre substratos de menor coste. Sin embargo, es posible que la aleación depositada no cuente con las propiedades tribológicas necesarias, por lo que es posible mejorarla mediante la adición de partículas cerámicas que le confieran un incremento de la resistencia al desgaste. La realización de compuestos cerámicos-metal mediante proyección térmica está bastante bien documentada en la bibliografía. De esta manera, se realizan recubrimientos de $\mathrm{NiCrBSi}$ para aplicaciones que soportan corrosión, temperatura y desgaste ${ }^{[16]}$. Las propiedades tribológicas del bronce pueden mejorarse utilizando partículas reforzantes de diamante utilizando la proyección térmica de llama ${ }^{[17]}$. También se ha publicado que mediante proyección fría se puede mejorar las propiedades antidesgaste del bronce utilizando partículas de diamante finamente envueltas por un film de níquel que favorece su deposición en el recubrimiento $^{[18]}$. Sin embargo, en algunos casos la utilización de una fase cerámica reforzante no mejora, sino al contrario disminuye las propiedades tribológicas del recubrimiento. De esta manera, la utilización de partículas de TiN en el interior de una matriz de bronce proyectado por proyección fría, aunque mejora su microdureza, disminuye su resistencia al desgaste ${ }^{[19]}$.

En el presente artículo se analiza el efecto de la adición de partículas reforzantes de alúmina sobre las propiedades del bronce de aluminio obtenido por proyección fría. Para ello se realiza una caracterización estructural de los recubrimientos, un estudio de la adherencia y microdureza de los compuestos obtenidos y finalmente un análisis de propiedades tribológicas entre las que se incluye ensayos de fricción, abrasión y erosión. 


\section{PROCEDIMIENTO EXPERIMENTAL}

Los polvos utilizados corresponden a un bronce de aluminio comercial Flame Spray Technologies M-950.32, manufacturado por atomización y una alúmina (corindón) Metco 105SFP producida por fundido y cuarteado. La composición nominal del polvo de bronce es, en peso, $89 \% \mathrm{Cu}, 10 \% \mathrm{Al}, 1 \% \mathrm{Fe}$, mientras que la alúmina tiene una pureza superior al 99,5 \%. La distribución del tamaño de partícula, medido mediante un difractómetro láser (Microtrac model X100/SRA 150) fue de $-53+12 \mu \mathrm{m}$ para el bronce de aluminio y $-31+4 \mu \mathrm{m}$ para la alúmina.

El substrato utilizado correspondía a un acero ferritico-perlítico $34 \mathrm{CrMo}_{4}$ con una microdureza de $\mathrm{HVN}_{100}=205$. Los recubrimientos se realizaron mediante un equipo de proyección fría Kinetics 4000 en el Centro de Proyección Térmica (CPT) de la Universidad de Barcelona. Las condiciones de proyección óptimas están indicadas en la tabla I.

Se ha partido de 4 polvos de proyección como mezcla mecánica de diferente porcentaje de bronce y alúmina (0\%, $15 \%$, $30 \%$ y $50 \%$ en volumen de alúmina). Se obtuvieron 4 recubrimientos designados PF - $0 \%$, PF - $3 \%$. PF - 7 \% y PF - $13 \%$, donde PF significa proyección fría y el número indica la proporción de alúmina depositada en el recubrimiento. Es importante constatar que la eficiencia de la deposición de la alúmina es muy inferior a la del bronce. Es por ello, que el contenido porcentual en volumen de la fase alúmina en el recubrimiento es bastante menor a la del polvo de partida. De esta manera, en la tabla II se observa que, a modo de ejemplo, el polvo con un contenido volumétrico del $50 \%$ de alúmina origina un recubrimiento con un contenido volumétrico del $13 \%$, es decir, que la $\mathrm{Al}_{2} \mathrm{O}_{3}$ tiene una deposición aproximadamente 4 veces inferior al bronce.

Los ensayos de microdureza se realizaron mediante un equipo Matzusawa MXT-OX a una carga de $100 \mathrm{~g}$ (dureza Vickers). El parámetro de rugosidad Ra (desviación estándar respecto a la media) se obtuvo como media de 5 medidas realizadas al azar en las muestras.

Los ensayos de fricción Ball-on-Disk se hicieron de acuerdo a la norma ASTM G99-03. Se utilizó una bola de WC - 6 \% Co de $11 \mathrm{~mm}$ de diámetro y $\mathrm{HVN}_{100}=1700$ como contraprobeta. Las condiciones de humedad y temperatura se mantuvieron constantes durante el ensayo e igual a $15-20 \%$ y $20^{\circ} \mathrm{C}$, respectivamente. Las probetas se desbastaron y pulieron hasta obtener una rugosidad de la probeta previo ensayo menor de $\mathrm{Ra}=0,2 \mu \mathrm{m}$. La distancia de deslizamiento fue $1000 \mathrm{~m}$, el diámetro de pista $16 \mathrm{~mm}$, velocidad de deslizamiento $0,11 \mathrm{~m} \cdot \mathrm{s}^{-1}$ y carga de $10 \mathrm{~N}$. Los caminos de desgaste se analizaron mediante SEM (Jeol JSM-5310) y el volumen perdido se analizó mediante interferometría de luz blanca, utilizando un programa que posibilitaba el cálculo de pérdida de volumen a través de imágenes en 3D (Zygo NewView

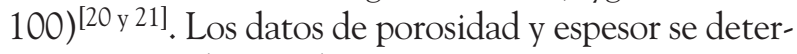
minaron mediante el software Matrox.

Los ensayos de abrasión se realizaron mediante un equipo Rubber Wheel, de acuerdo con la norma ASTM G65-00, utilizando un caudal de arena abrasiva de $\mathrm{SiO}_{2}$ constante de tamaño de partícula de $0,4 \mathrm{~mm}-0,8 \mathrm{~mm}$ y un giro de la rueda de $131 \mathrm{rev} \cdot \mathrm{min}^{-1}$.

Los ensayos de erosión se realizaron con un equipo convencional de granallado con un flujo fijo de arena erosiva de $\mathrm{Al}_{2} \mathrm{O}_{3}$ de tamaño de partícula $0,1 \mathrm{~mm}$ $0,2 \mathrm{~mm}, 90^{\circ}$ de ángulo de impacto y distancia entre la boquilla y la muestra de $20 \mathrm{~cm}$, con una presión de gas de 2,5 bar. Los ensayos de adherencia se determinaron de acuerdo a la norma ASTM C633-01.

\section{RESULTADOS Y DISCUSIÓN}

\subsection{Caracterización estructural}

La estructura representativa de los polvos utilizados se muestra en las figuras 2 y 3 . Se aprecia la forma angulosa de la alúmina y la morfología redondeada de las partículas de bronce de aluminio.

Tabla I. Condiciones de proyección óptimas de los recubrimientos

Table I. Optimal spraying conditions of the coatings

\begin{tabular}{lc}
\hline Presión de gas (bar) & $32-40$ \\
Temperatura de gas $(\mathrm{K})$ & $800-1100$ \\
Distancia de proyección $(\mathrm{mm})$ & 40 \\
Velocidad transversal de la pistola $(\mathrm{mm} / \mathrm{s})$ & 500 \\
Capas depositadas & 3 \\
Capas de granallado & 2
\end{tabular}


Tabla II. Características de los recubrimientos Valores indicados como media y desviación estándar

Table II. Main features of the coatings. Average value and standard deviation are indicated

\begin{tabular}{lcccc}
\hline Denominación & PF - 0 \% & PF - 3 \% & PF - 7 \% & PF - 13 \% \\
\hline Contenido de $\mathrm{Al}_{2} \mathrm{O}_{3}$ en polvo de partida $(\% \mathrm{vol})$ & 0 & 15 & 30 & 50 \\
Contenido de $\mathrm{Al}_{2} \mathrm{O}_{3}$ en recubrimiento $(\% \mathrm{vol})$ & 0 & 3 & 7 & 13 \\
Espesor $(\mu \mathrm{m})$ & $632 \pm 55$ & $590 \pm 40$ & $482 \pm 15$ & $374 \pm 11$ \\
$\mathrm{Ra}(\mu \mathrm{m})$ & $12,5 \pm 2,1$ & $13,0 \pm 1,8$ & $11,9 \pm 1,2$ & $12,3 \pm 2,0$ \\
Microdureza $(\mathrm{HVN} 100)$ & $248 \pm 29$ & $260 \pm 13$ & $262 \pm 29$ & $286 \pm 21$ \\
Adherencia $(\mathrm{MPa})$ & 20 & 22 & 25 & 44 \\
\hline
\end{tabular}

A partir de las mezclas mecánicas de diferentes proporciones de bronce y alúmina se realizaron los recubrimientos mediante proyección fría. Cabe destacar que inicialmente se llevaron a cabo diferentes pruebas de proyección previas modificando los parámetros distancia de proyección, temperatura de proyección, presión del gas de trabajo y caudal de alimentación para finalmente obtener las condiciones de proyección adecuadas.

Es importante destacar que, aunque en muchos casos no hace falta granallar el substrato para hacer recubrimientos mediante la técnica de proyección fría, en el presente estudio se realizó un granallado previa deposición con alúmina. Se utilizó el mismo equipo de proyección, haciendo circular polvo de alúmina a una presión de 20 bar, distancia de $40 \mathrm{~mm}$ y temperatura ambiente. La alúmina se deposita sobre el substrato, sin embargo, estudios internos indican que en esta técnica este fenómeno no tiene

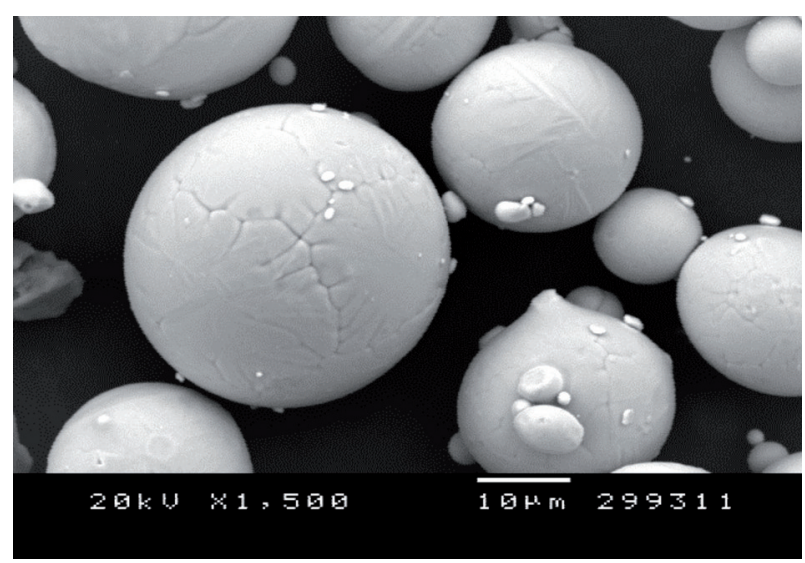

Figura 2. Morfología del polvo de bronce de aluminio de partida.

Figure 2. Morphology of the initial aluminumbronze powder. un efecto negativo sobre la adherencia del recubrimiento.

Los recubrimientos obtenidos se muestran en las figuras 4 y 7 . La figura 4 , correspondiente a bronce de aluminio sin adición de alúmina (PF - $0 \%$ ) presenta una estructura densa con buena adherencia al substrato ( $\sin$ poros ni grietas en la interfase) e inexistencia de óxidos. El recubrimiento consta de 2 zonas claras, la superior donde existe una porosidad evidente y la inferior totalmente densa. Esto se debe al proceso de constitución del recubrimiento en sí y no tiene una repercusión negativa sobre la vida útil de la pieza ya que normalmente la zona porosa se eliminaría por mecanizado para dar a la pieza las dimensiones correctas (Fig. 8). En la proyección fría, las partículas no fundidas del polvo de proyección van impactando sobre el recubrimiento previamente depositado. Este efecto shot-peening se manifiesta en dos resultados principales, el endurecimiento de

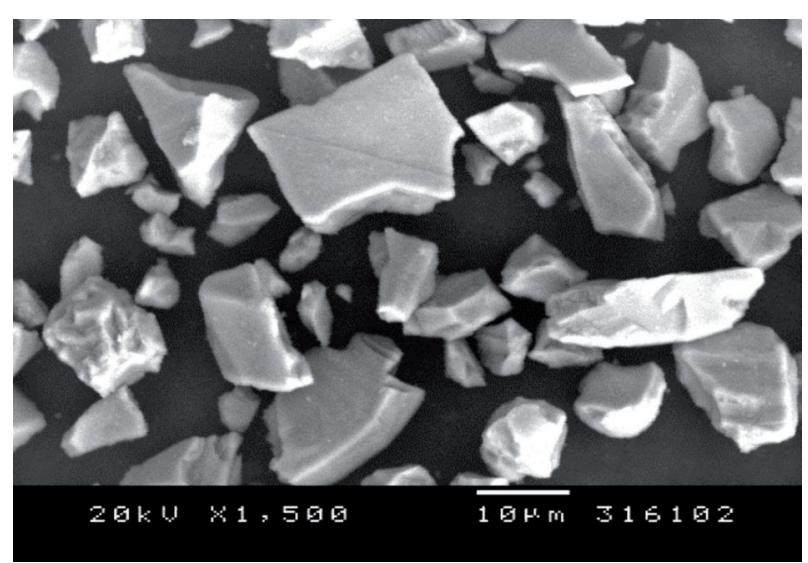

Figura 3. Morfología del polvo de alúmina de partida.

Figure 3. Morphology of the initial alumina powder. 


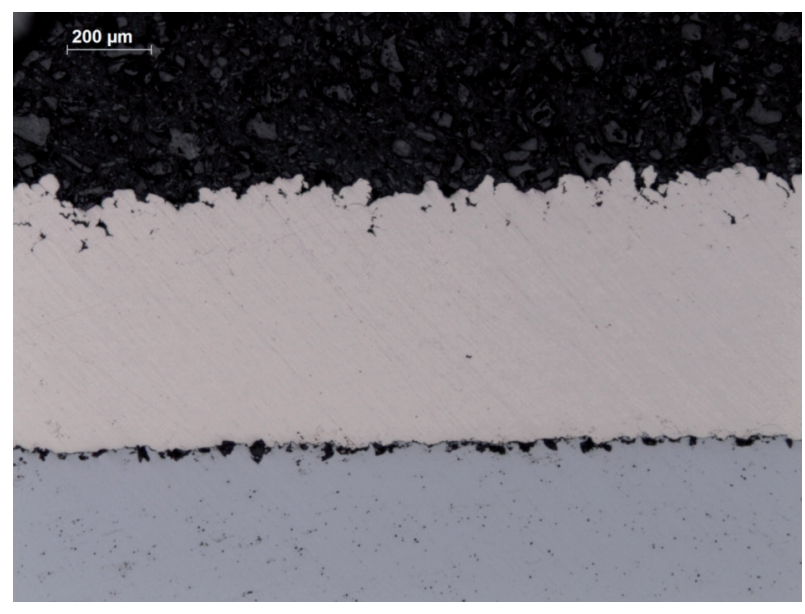

Figura 4. Recubrimiento PF - $0 \%$. Nótese la porosidad solo en zona más externa y ausencia de óxidos. La interfase substrato-recubrimiento muestra partículas de alúmina provenientes del proceso de granallado.

Figure 4. PF - $0 \%$ coating. Note the porosity in the outer side and lack of oxides. Interface substrate-coating show alumina particles from the grit-blasting process.

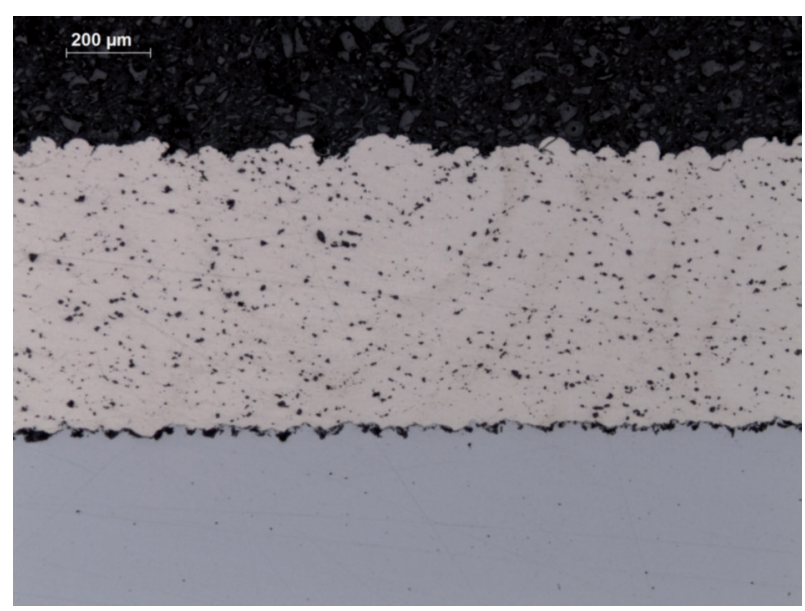

Figura 5. Recubrimiento PF - $3 \%$. La alúmina se encuentra perfectamente distribuida en el interior del recubrimiento. No hay porosidad visible con SEM.

Figure 5. PF - $3 \%$ coating. Alumina (dark particles) is perfectly distributed in the coating. There is no porosity shown with SEM.

las partículas depositadas y la disminución de porosidad del recubrimiento previamente depositado.

La figura 9 muestra una imagen de la estructura del recubrimiento atacada mediante $\mathrm{FeCl}_{3}$ disuelto

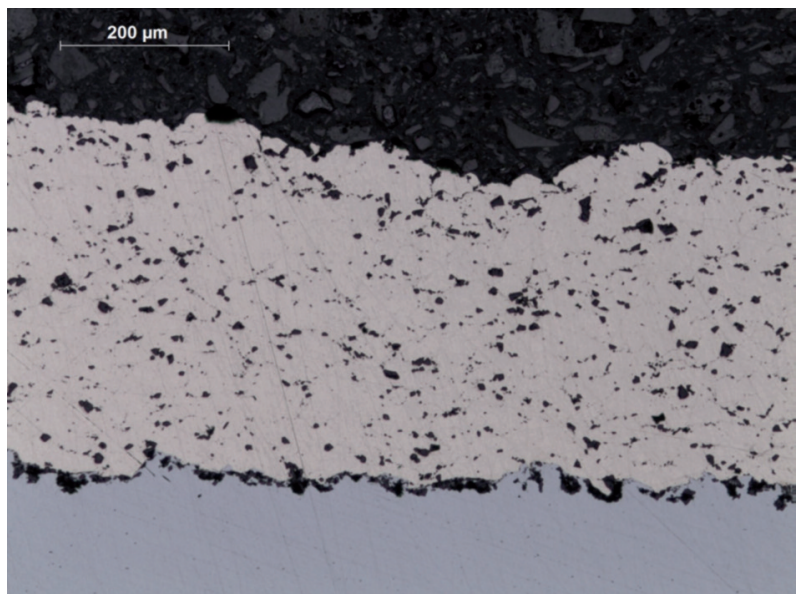

Figura 6. Recubrimiento PF - $7 \%$. Recubrimiento con un $7 \%$ de alúmina.

Figure 6. PF - $7 \%$ coating. Coating with $7 \%$ of alumina.

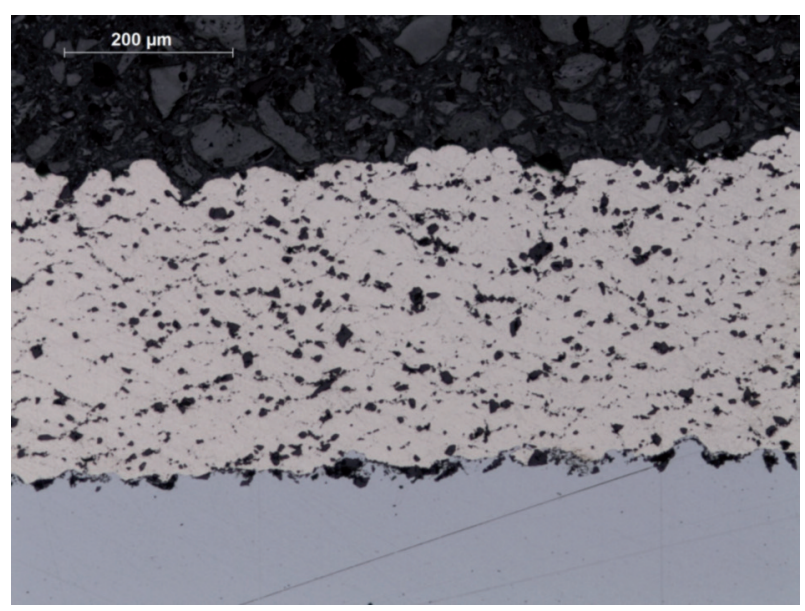

Figura 7. Recubrimiento PF - $13 \%$. Recubrimiento muy denso y sin grietas. La fase oscura corresponde solamente a alúmina.

Figure 7. PF - $13 \%$ coating. Dense coating without cracks. Dark phase is only alumina.

en una mezcla de $\mathrm{HCl}$ y etanol. Se observa que el exterior de las partículas presenta una deformación evidente de la estructura debido al impacto a altas velocidades durante el proceso de constitución del recubrimiento. Estudios llevados a cabo en el Centro de Proyección Térmica han desvelado que este proceso de deformación da lugar a valores de microdureza muy superiores a los recubrimientos obtenidos por técnicas en las que se funde el material depositado, como es el caso de la proyección de plasma atmosférico. De esta manera, y a modo indicativo, 


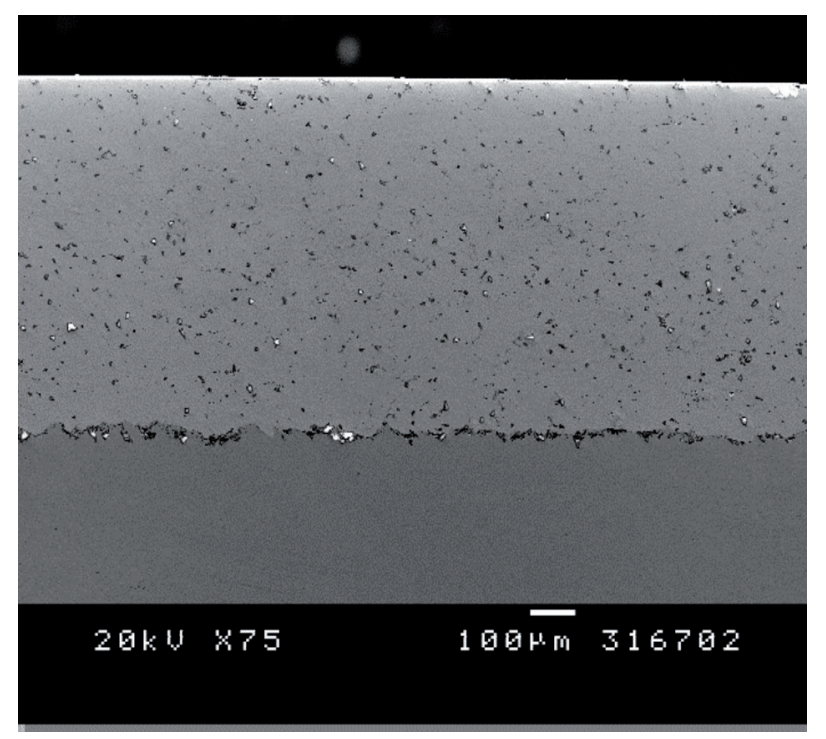

Figura 8. Imagen de la pieza mecanizada PF $3 \%$.

Figure 8. Image of the sample PF - $3 \%$ after machining.

el valor del recubrimiento de bronce obtenido por plasma es de $\mathrm{HVN}_{100}=157$ frente a $\operatorname{los} \mathrm{HVN}_{100}=248$ del recubrimiento obtenido por proyección fría.

El análisis de Difracción de Rayos X del recubrimiento de bronce y polvo inicial de bronce muestra que se trata de una estructura bifásica $\left(\mathrm{Cu}\right.$ y $\left.\mathrm{Cu}_{3} \mathrm{Al}\right)$ sin observarse la presencia de fases amorfas (Figs. 10 (a) y 10 (b) ). Un análisis detallado de la posición de los picos permite ver que existen pequeños desplazamientos del orden de la décima de grado en los picos del recubrimiento atribuibles a las deformaciones de la red cristalina que se producen debido a las altas tensiones residuales de compresión existentes en el recubrimiento como consecuencia del impacto a altísima velocidad de las partículas (efecto shot-peening) sobre el material predepositado.

Los recubrimientos compuestos bronce-alúmina presentan una estructura densa y una buena cohesión entre las partículas reforzantes de alúmina y la matriz de bronce (Fig. 11). Es por ello que no se observa microporosidades entre estas dos fases, sino todo al contrario, una excelente unión entre partículas cerámico-metal. El análisis microscópico de la superficie libre de los recubrimientos permite constatar que están constituidos por un conjunto de partículas de forma redondeada (similares al polvo de partida) de bronce que se han deformado durante su impacto en el recubrimiento previamente depositado, así como partículas angulosas de alúmina que deforman fuertemente el bronce pre-depositado y mantienen inalterada su morfología superficial (Fig. 12).

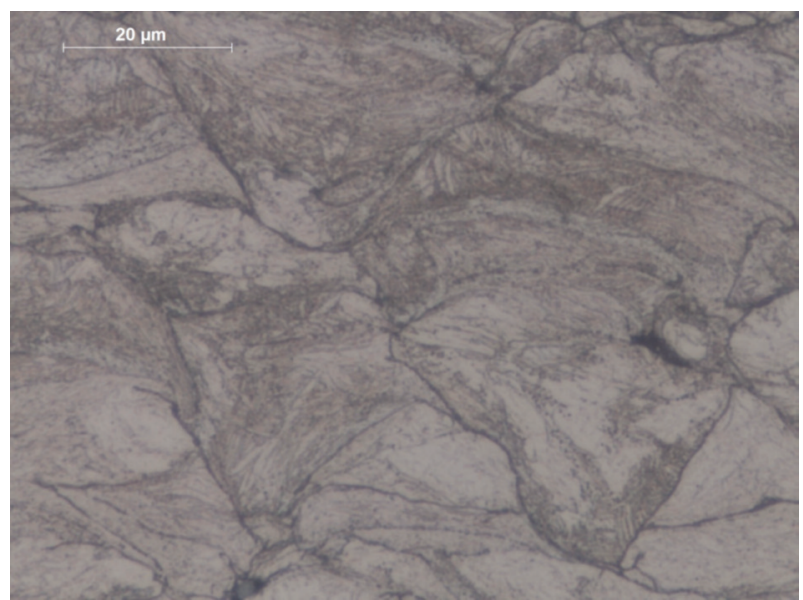

Figura 9. Estructura atacada del recubrimiento de bronce, en la que se observa la perfecta acomodación de las diferentes partículas que constituyen el recubrimiento.

Figure 9. Etched structure of the bronze coating, showing the perfect accommodation of the particles that form the coating.

Es posible constatar que existe un gran número de pequeñas partículas cerámicas de tamaño muy fino (por debajo de $1 \mu \mathrm{m}$ ) en el recubrimiento, que no aparecían en el polvo original. Durante el proceso de deposición, las partículas cerámicas chocan en el haz de proyección entre sí, rompiéndose y generando otras partículas de menor tamaño que se depositan en el recubrimiento. Este efecto es tanto más grande cuanto mayor sea el porcentaje de fase cerámica en el polvo de partida. El parámetro de rugosidad Ra en los recubrimientos es prácticamente constante por lo que no existe diferencia en la morfología de su superficie libre, manteniéndose una diferencia máxima de alrededor del $10 \%$ (Tabla II).

\subsection{Propiedades mecánicas y tribológicas}

Los valores de microdureza y adherencia de los recubrimientos se muestran en la tabla II. Es importante destacar que la microdureza de los depósitos se incrementa con el contenido de alúmina; así una cantidad de $13 \%$ de fase reforzante en el depósito aumenta un $15 \%$ la dureza del mismo. Por otro lado la adherencia también aumenta con el contenido de alúmina debido al efecto de granallado que esta fase dura origina sobre la superficie del substrato a la presión de proyección, que además de dotarle de una rugosidad, elimina los restos de óxidos y residuos depositados que jugarían un papel negativo en la adherencia con el recubrimiento. 


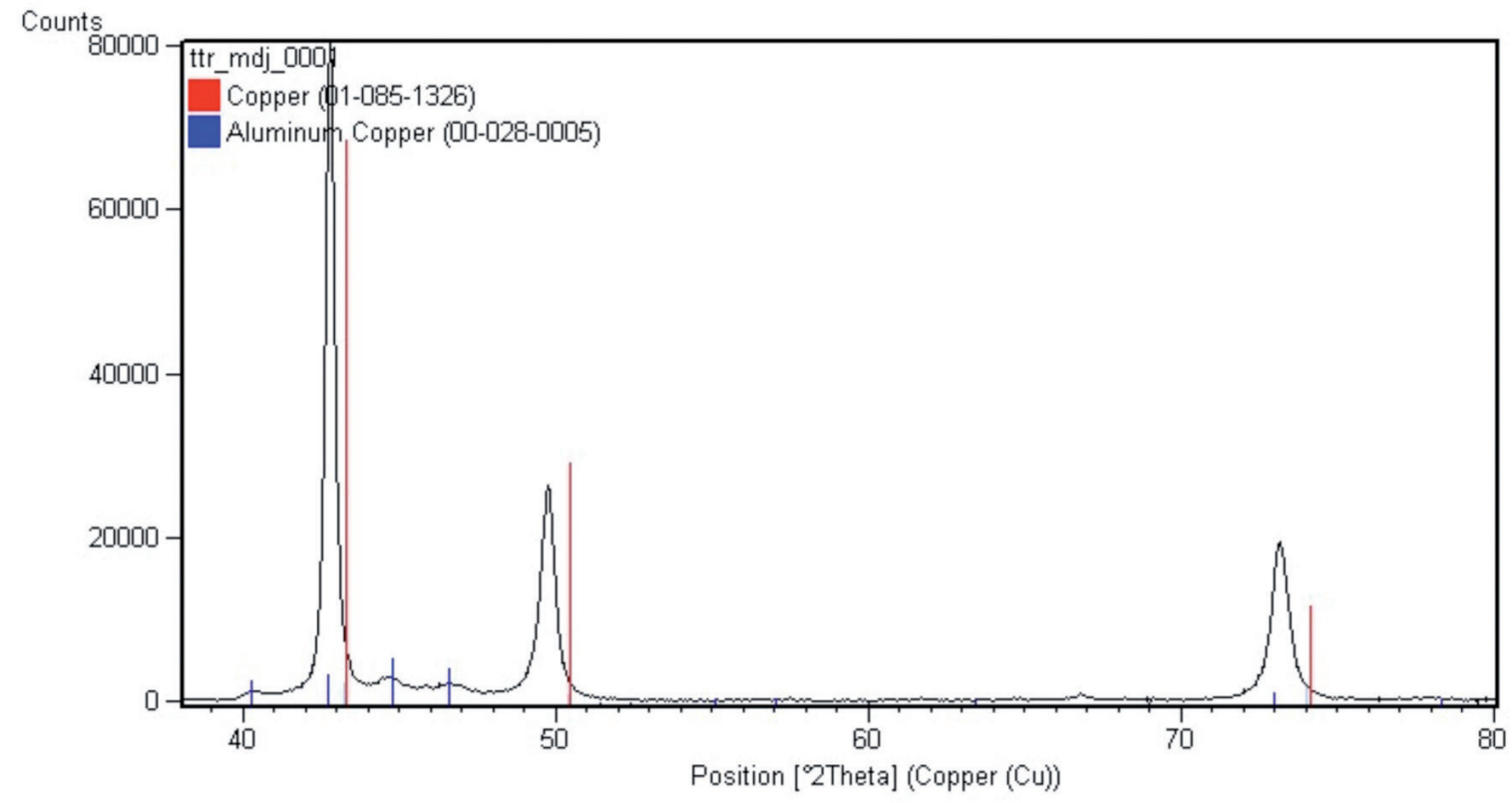

(a)

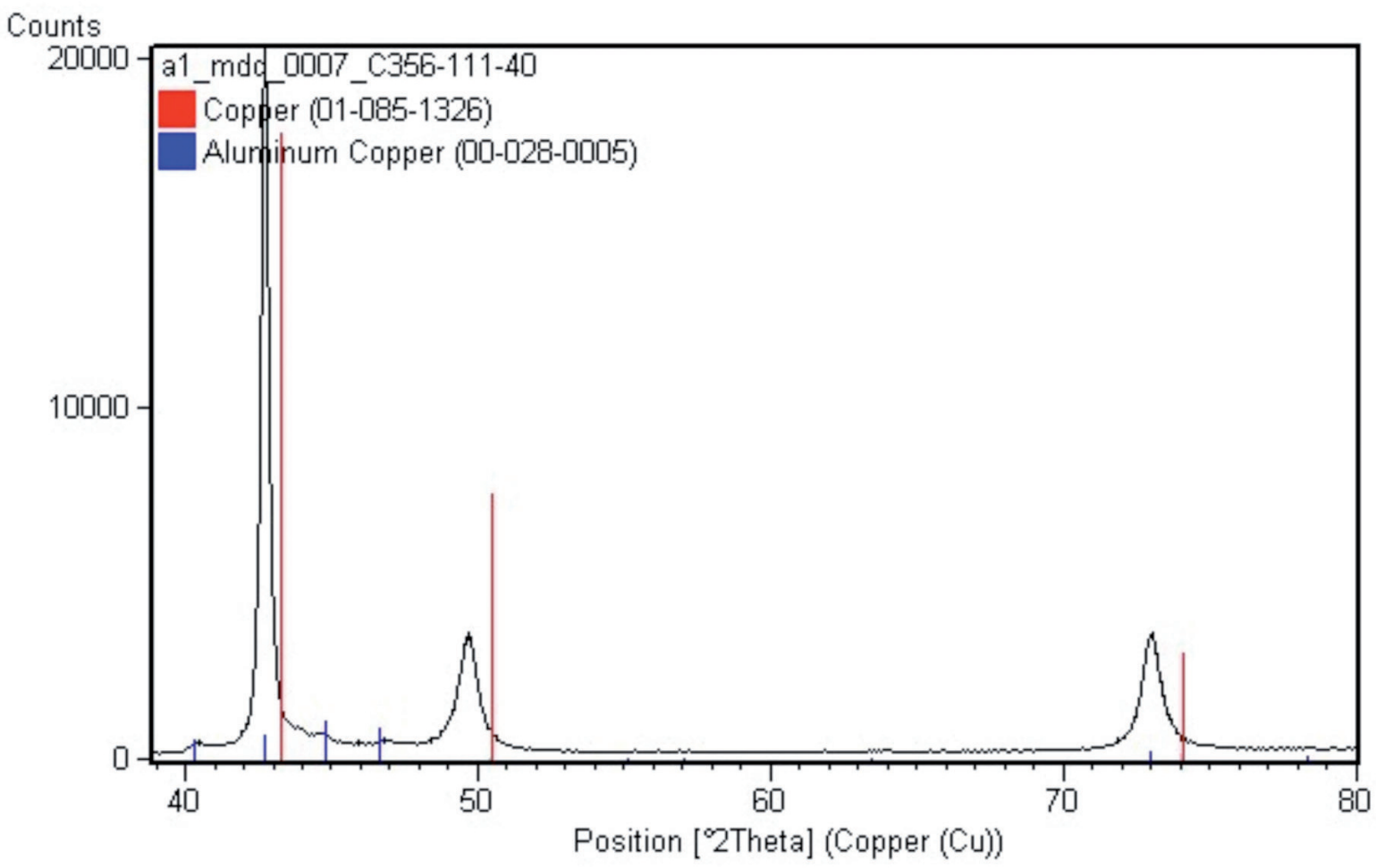

(b)

Figura 10. (a) Difractograma del polvo original de bronce, constituido por las fases $\mathrm{Cu}$ (solución sólida) y $\mathrm{Cu}_{3} \mathrm{Al}$; (b) Difractograma del recubrimiento de bronce, constituido por $\mathrm{Cu}$ (solución sólida) y $\mathrm{Cu}_{3} \mathrm{Al}$.

Figure 10. (a) XRD image of the initial bronze powder, composed by $\mathrm{Cu}$ (solid solution) and $\mathrm{Cu}_{3} \mathrm{Al}$; (b) XRD image of the bronze coating, composed by $\mathrm{Cu}$ (solid solution) and $\mathrm{Cu}_{3} \mathrm{Al}$. 


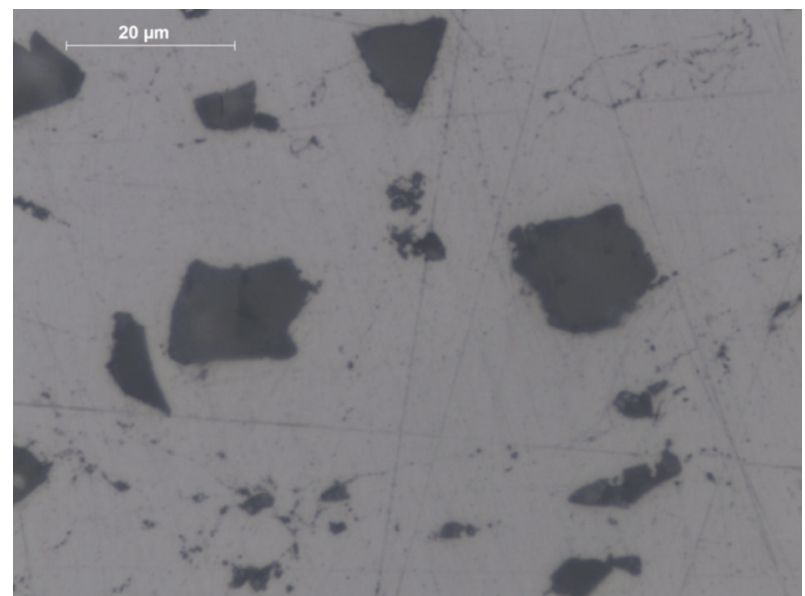

Figura 11. Imagen de la perfecta cohesión entre partículas de bronce y alúmina y presencia de finísimas partículas de la fase cerámica fracturadas durante la proyección (notar que no se trata de porosidad).

Figure 11. Image of the perfect cohesion between particles of bronze and alumina and the finely distributed ceramic phase broken during the spraying (note that it is not porosity).

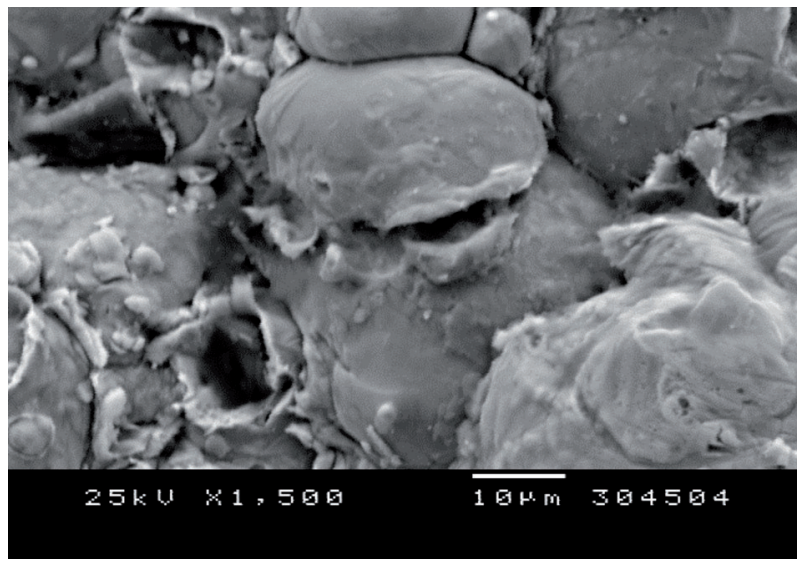

Figura 12. Superficie libre del recubrimiento. Se observan partículas de alúmina incrustadas en la matriz de bronce.

Figure 12. Surface of the coating. Note the alumina particles in the bronze matrix.

— Vol. Perdido (mm3)

$\square$ Coef. Fricciór $(\boldsymbol{\mu})$

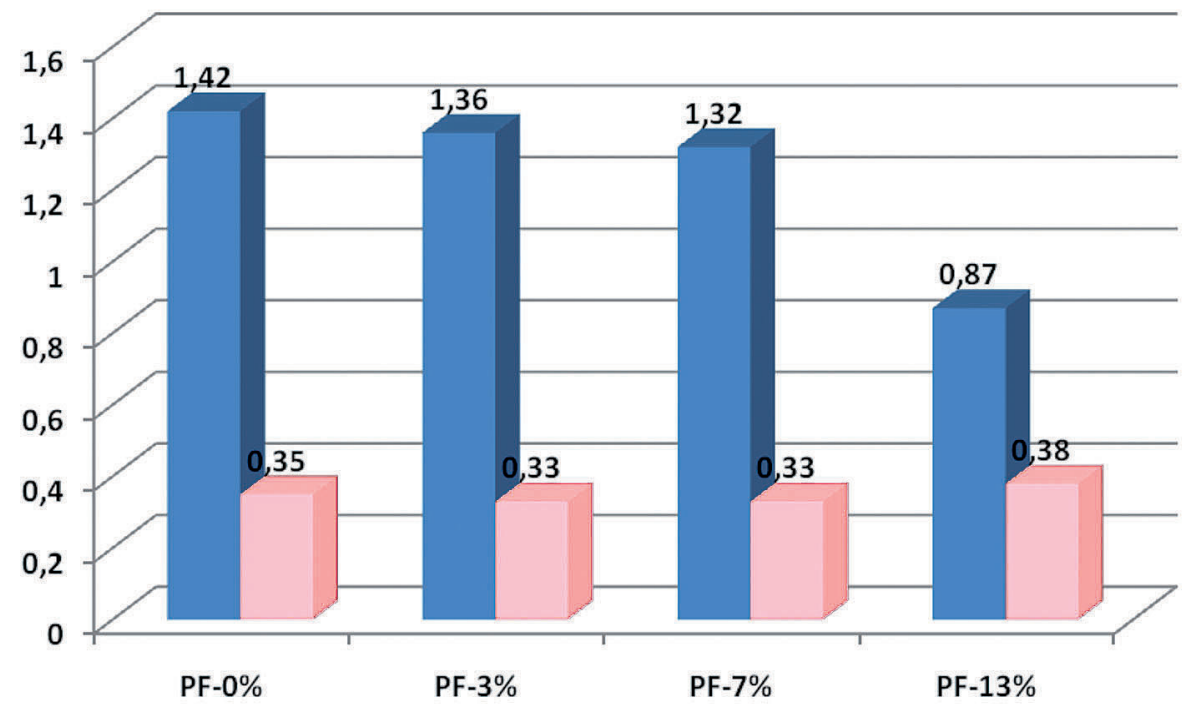

Figura 13. Volumen perdido y coeficiente de fricción de los recubrimientos tras el ensayo Ball-on-Disk.

Figure 13. Volume loss and friction coefficient for coatings after the Ball-on-Disk test. 


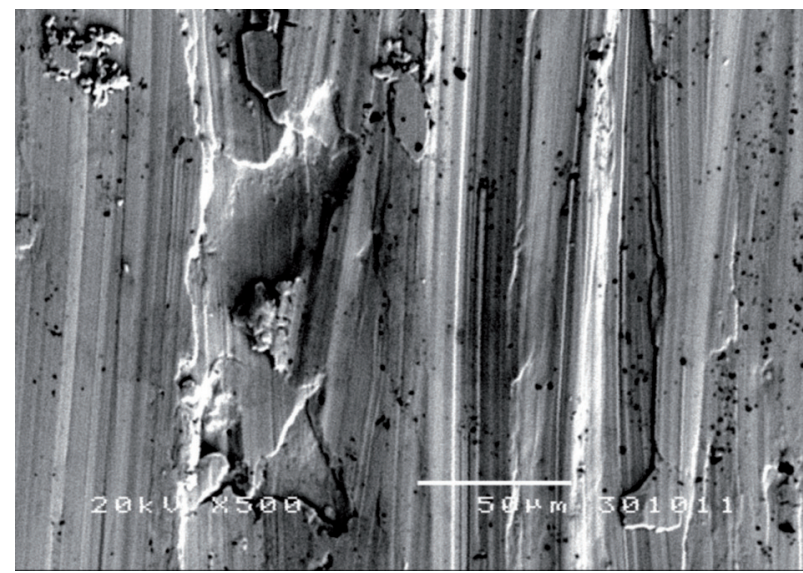

Figure 14. Camino de desgaste del recubrimiento PF - $3 \%$ después del ensayo Ball on Disk.

Figure 14. Wear track of the PF - $3 \%$ after the Ball on Disk test.

La figura 13 presenta los valores de desgaste y coeficiente de fricción de los recubrimientos frente a la bola de WC - 6 \% Co tras el ensayo Ball-on-Disk. Se observa una clara dependencia del desgaste con la proporción de fase cerámica. De esta manera, un contenido del $13 \%$ en alúmina origina un volumen de la huella cercano a la mitad del que ocurre en el depósito de bronce puro. El coeficiente de fricción no se ve afectado por el cambio de composición del depósito y los valores se sitúan entre $\mu=0,3$ y $\mu=0,4$. Estos valores, relativamente bajos, hacen pensar en su posible aplicación en útiles sometidos a fricción, donde se desee coeficientes bajos y una resistencia al desgaste moderada. El camino de desgaste tras el ensayo presenta una baja cantidad de detritus adherido y su superficie libre presenta un rayado paralelo como consecuencia de la abrasión de las partículas de WC y del material que se ha desgastado del propio recubrimiento siguiendo un proceso de abrasión de 3 cuerpos (Fig. 14). Podemos concluir, según las observaciones indicadas anteriormente, que el principal mecanismo de desgaste que sufren los recubrimientos ante la situación de fricción es la abrasión. Este proceso está originado tanto por la abrasión ocasionada por la contraprobeta debido a su alta dureza (alrededor de $\mathrm{HVN}_{100}=1700$ ), como por el mismo detritus generado durante el proceso de fricción y que contiene partículas mayormente duras abrasivas provenientes de la contraprobeta (óxidos de aluminio y cobre). Otros mecanismos, como la delaminación entre partículas, aunque podrían estar presentes, no se observan mediante el análisis superficial de la superficie del camino de desgaste.

La resistencia de los recubrimientos ante el desgaste abrasivo se muestra en la figura 15 (ensayo Rubber Wheel). Después de unos valores inicialmente altos, como consecuencia de la eliminación de la rugosidad inicial, la velocidad de desgaste se

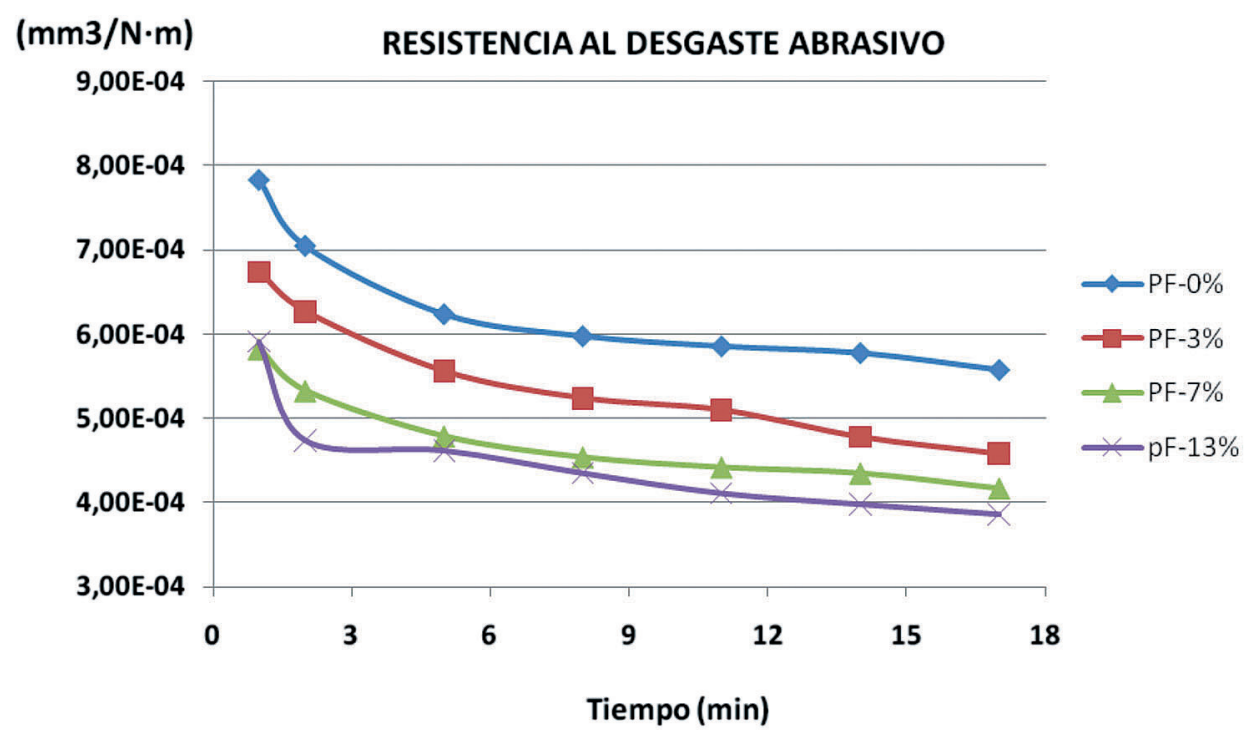

Figura 15. Evolución de la resistencia al desgaste abrasivo para los diferentes recubrimientos.

Figure 15. Evolution in the abrasive wear resistance of the different coatings 


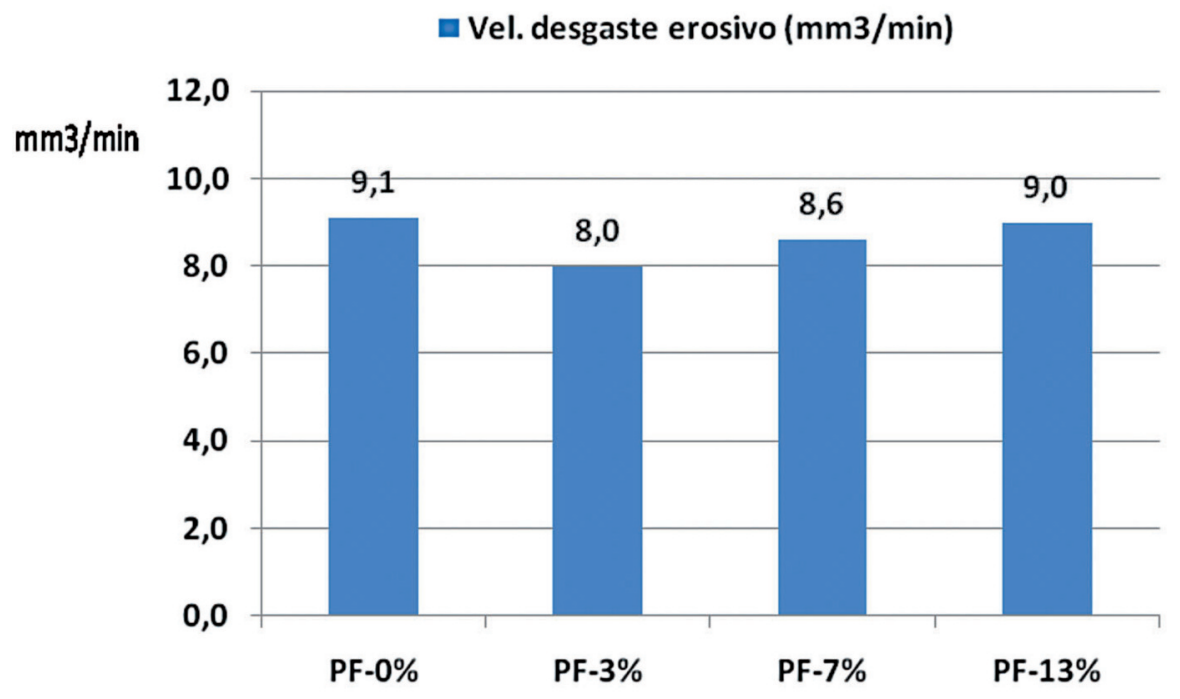

Figura 16. Velocidad de desgaste erosivo de los recubrimientos.

Figure 16. Erosive wear rate of coatings.

estabiliza. Los datos indican una mejora del comportamiento con la adición de alúmina, alcanzando valores de velocidad de abrasión $30 \%$ inferiores cuando el recubrimiento depositado contiene $13 \%$ de fase cerámica reforzante. La fase cerámica alúmina, situada en forma de partículas discretas distribuidas homogéneamente en los composites bronce-alúmina, impide de forma muy efectiva el desgaste por el agente abrasivo (óxido de silicio) y le confiere una mejora en su resistencia ante el desgaste.

Los resultados de velocidad de desgaste erosivo se encuentran en la figura 16. Es bien conocido que la resistencia a este tipo de desgaste depende del ángulo de incidencia de las partículas, siendo especialmente agresivo a $90^{\circ}$ para materiales cerámicos puesto que la energía de impacto de las partículas es mayor y su capacidad de deterioro debido a su alta fragilidad es superior. Sin embargo, vemos que los valores de velocidad de desgaste son prácticamente constantes independientemente del contenido de fase cerámica. Esto se debe a que la alúmina se encuentra perfectamente envuelta de matriz metálica tenaz, por lo que la energía de las partículas erosivas puede absorberse perfectamente por esta matriz mediante su deformación plástica.

\section{CONCLUSIONES}

- La proyección fría es una técnica reciente de la familia de la proyección térmica que permite la obtención de recubrimientos de altí- sima densidad y sin degradación (ya sea oxidación o disolución de partículas) durante su procesado.

- Los recubrimientos de bronce de aluminio son muy compactos, con una porosidad en su zona densa imperceptible, y su valor de dureza superior a los obtenidos por proyección plasma debido a los procesos de acritud que tienen lugar a causa del fuerte impacto que se produce durante su formación. La adherencia con el substrato se incrementa en los recubrimientos compuesto debido al efecto granallado que producen las partículas cerámicas.

- La adición de alúmina permite incrementar los valores de resistencia a la abrasión y a la fricción, sin que se vea afectado su comportamiento ante la erosión. De esta manera se obtienen resistencias al desgaste por deslizamiento cercanas al $100 \%$ superiores a la del bronce puro, mientras que la resistencia por abrasión mejora en más de un $30 \%$.

- El mecanismo principal de desgaste durante el proceso de fricción es la abrasión que tiene lugar tanto por la contraprobeta de WC - $6 \%$ Co como por las partículas abrasivas generadas tras el desgaste del propio recubrimiento (detritus). Los resultados de resistencia a la erosión de los composites bronce-alumina también son satisfactorios a pesar de tener hasta un $13 \%$ de fase cerámica, debido a que la matriz metálica de bronce absorbe perfectamente la energía de las partí- 
culas erosivas y puede deformarse plásticamente, sin que exista una pérdida importante de recubrimiento.

- Estos resultados abren futuras perspectivas frente a la consecución de depósitos de materiales compuestos metal-cerámicos compactos de espesores variables, recubrimientos de materiales sin degradación estructural con relación al polvo original y con propiedades tribológicas muy mejoradas.

\section{Agradecimientos}

Los autores agradecen a la Generalitat de Catalunya y al Ministerio de Ciencia e Innovación el soporte económico en los proyectos SGR2009-390 y MAT2010-20311.

\section{REFERENCIAS}

[1] S.H. Zahiri, W. Yang y M .Jahedi, J. Therm. Spray Techn. 18 (2009) 110-117.

[2] T.S. Price, P.H. Shipway y D.G. McCartney, J. Therm. Spray Techn. 20515 (2006) 507-512.

[3] N. Cinca, M. Barbosa, S. Dosta y J.M. Guilemany, Surf. Coat. Tech. (2010) 1.0961.102.

[4] H. Koivuluoto, M. Honkanen y P. Vuoristo, Surf. Coat. Tech. 204 (2010) 2.353-2.361.

[5] R. Ghelichi y M. Guagliano, Frattura et Integrità Strutturale 8 (2009) 30-44.

[6] T. Schmidt, F. Gärtner, H. Assadi y H. Kreye, Acta Mater. 54 (2006) 729-742.
[7] R. Kingswell, D.S. Rickerby, S.J. Bull y K.T. Scott, Thin Solid Films 198 (1991) 139-148.

[8] H.J. Kim, C.H. Lee y S.Y. Hwang, Mat. Sci. Eng. A 391 (2005) 243-248.

[9] A. Papyring, V. Kosarev, S. Klinkov, A. Alkimov y V. Fomin, Cold Spray Technology, Ed. Elsevier, Oxford, England, 2007, pp. 248323.

[10] H.J. Kim, C.H. Lee y S.Y. Hwang, Surf. Coat. Tech 191 (2005) 335-340.

[11] S.H. Zahiri, C.I. Antonio y M. Jahedi, J. Mater. Process. Technol. 209 (2009) 922-929.

[12] J.M. Guilemany, J. Nin, C. Lorenzana, J.M. Miguel y J.R. Miguel, Bol. Soc. Esp. Ceram. V. 43 (2004) 483-487.

[13] Z.Z. li, L.D. Yuan y W.S. Yong, Trans. Nonferreous Met. Soc. China 16 (2006) 868872.

[14] J.M. Tura, A. Traveria, M.D. de Castellar y J. Pujadas, Wear 189 (1995) 70-76.

[15] D.V. Kudashov, R. Zauter y H.R. Müller, Mat. Sci. Eng. A 477 (2008) 43-49.

[16] J.M. Guilemany, J.M. Miguel y S. Vizcaino, Tribol. Int. 36 (2003) 181-187

[17] K. Venkateswarlu, V. Rajinikanth, T. Naveen, D.P. Sinha, Atiquzzaman y A.K. Ray, Wear 266 (2009) 995-1.002.

[18] H. Na, G. Bae, S. Shin, S. Kumar, H. Kim y C. Lee, Compos. Sci. Technol. 69 (2009) 463-468.

[19] X. Guo, G. Zhang, W. Li, Y. Gao, H. Liao y C. Coddet, Appl. Surf. Sci. 255 (2009) 3.822-3.828.

[20] J.M. Guilemany, J.M. Miguel, S. Armada, S. Vizcaino y F. Climent, Mater. Charact. 47 (2001) 307-314.

[21] J.M. Guilemany, S. Armada y J.M. Miguel, J. Therm. Spray Techn. 10 (2001) 142-146. 